\title{
Bacteremia with Cutaneous Nodules, Due to Pseudomonas Aeruginosa
}

\author{
Ali Akbar Heydari', Maryam Mojtabavi ${ }^{2}$ \\ ${ }^{1}$ Imam Reza Hospital, Mashhad University of Medical Sciences, Mashhad, Iran; ${ }^{2}$ Mashhad University of Medical Sciences, Mashhad, \\ Iran. \\ Email: Heydariaa@mums.ac.ir
}

Received November $1^{\text {st }}$, 2011; revised December $2^{\text {nd }}, 2011$; accepted December $10^{\text {th }}, 2011$.

\begin{abstract}
Pseudomonas aeruginosa bacteremia rarely occurs in non-immunocompromised adults and can be difficult to be treated. We report a case of 35-year-old woman who presented with respiratory distress, fever and skin lesions. There was pleural effusion in chest radiograph, and CT scan of thorax showed necrotizing pneumonia and loculated empyema. Among the cultures of blood, empyema, sputum and the skin nodules aspirates, all were positive for Pseudomonas aeruginosa. The patient was treated with ciprofloxacin, amikacin and then pleural decortications. Laboratory tests failed to reveal any immunological deficits. After a period of 21 days of antibiotic therapy, the patient was discharged from hospital with a good condition.
\end{abstract}

Keywords: Pseudomonas, Aeruginosa, Bacteremia, Cutaneous Nodules.

\section{Introduction}

Pseudomonas aeruginosa (PA) is an opportunistic pathogen which rarely causes a disease in healthy persons, either children or adults [1-3]. These infections often occur in patients with underlying conditions. Our patient presented with Pseudomonas aeruginosa bacteremia, empyma, lung abscess and the rare form of metastatic lesion (skin nodules).

\section{Case Presentation}

A 35-years-old female was admitted to the Infectious Diseases department, for respiratory distress. The illness had begun 18 days earlier with abdominal pain in association with pleuretic-type flank pain, which were accompanied by fever and rigors. Following aggravation of the abdominal pain, laparotomy was performed with the probable diagnosis of appendicitis, but there were no abnormal findings in the abdomen. Her fever was continued despite antimicrobial therapy and her condition was worsened progressively. She was ill and her extremities were cyanotic and there were multiple subcutaneous scalded nodules (Figure 1) on her limbs.

There was no history of previous disease including human immunodeficiency virus infection.

High resolution computed tomography of the chest was performed, and it showed an abscess in apical-posterior

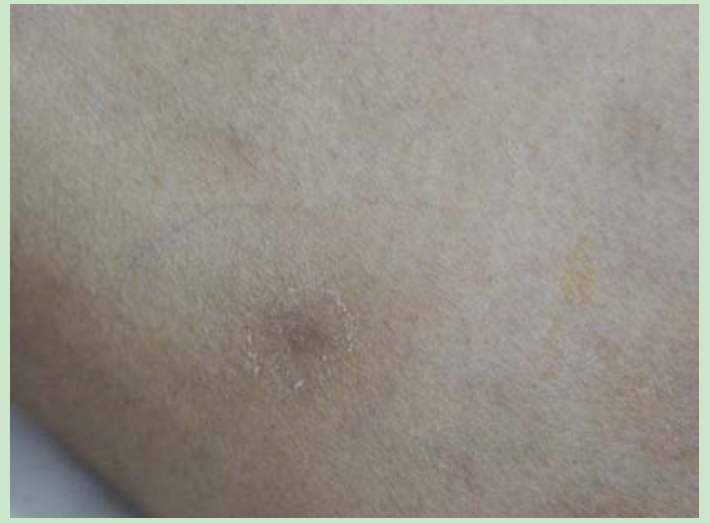

Figure 1. Subcutaneous nodule in a patient with pseudomonas aeroginosa bacteremia.

segment of right lower lobe, with louculated pleural effusion. Among the cultures of blood, empeyma, sputum, and the skin nodules aspirates, all were positive for pseudomonas aeruginosa.

\section{Discussion}

The only point differentiating this entity from other causes of gram negative sepsis is the skin lesion known as ecthyma gangrenosum (EG). Infection with the bacteria should be suspected in the patients who presented with EG but this lesion is not pathognomonic. 
Howevere other skin lesions may occasionally accompany with pseudomonal bacteremia, such as subcutaneous nodules [4]. Other cutaneous lesions that occur during bacterial septicemia include: maculopapular lesions vesicles or pustules, and cellulitis, [5,6]. Our patient presented with Pseudomonas aeruginosa bacteremia, empyma, lung abscess and the rare form of metastatic lesion, skin nodules. One may correctly suspect that the patient's laparotomy was the source of the pseudomonas entry, but there was a pleuretic-type flank pain before surgery suggesting that a probable pseudomonal empyema already occurred earlier.

\section{Conclusions}

The presence of the skin nodules as metastatic lesions of pseudomonal bacteremia along with bacteremia and severe pleuropulmonary infection in an immunocompetent young patient is a rare event. The clinicians should be aware of this clinical picture and even in typical patients, and search for unusual pathogens regardless of patients' immunologic status.

\section{REFERENCES}

[1] P. D. Kumar, K. Ravakhah and B. C. West, "Dissemi- nated Pseudomonas Aeruginosa and Necrotizing Pneumonia with Complete Recovery," Southern Medical Journal, Vol. 94, No. 2, 2001, pp. 229-232.

[2] S. Ishihara, M. Takino, Y. Okada and K. Mimura, "Septic Shock Due to Pseudomonas Aeruginosa in a Previously Healthy Woman,” Intensive Care Medicine, Vol. 21, No. 3, 1995, pp. 226-228. doi:10.1007/BF01701476

[3] C. C. Mull, R. J. Scarfone and D. Conway, "Ecthyma Gangrenosum as a Manifestation of Pseudomonas Sepsis in a Previously Healthy Child," Annals of Emergency Medicine, Vol. 36, No. 4, 2000, pp. 383-387. doi:10.1067/mem.2000.110013

[4] D. Diaz-Valle, J. M. Benitez del Castillo, M. J. Fernandez Acenero, et al., "Endogenous Pseudomonas Endophthalmitis in an Immunocompetent Patient,” European Journal of Ophthalmology, Vol. 17, No. 3, 2007, pp. 461-463.

[5] R. Roberts, M. M. Tarpay, M. I. Marks and R. Nitschke, "Erysipelas Like Lesions and Hyperesthesia as Manifestations of Pseudomonas Aeruginosa Sepsis," Journal of the American Medical Association, Vol. 248, No. 17, 1982, p. 2156. doi:10.1001/jama.1982.03330170060030

[6] C. Jr. Forkner, E. Frel Jh and U. Jp Edgcomb, "Pseudomonas Septicemia, Observations on Twenty-Three Cases,” American Journal of Medicine, Vol. 25, No. 6, 1958, p. 877. doi:10.1016/0002-9343(58)90060-3 